\title{
Investigating the molecular basis of local adaptation to thermal stress: population differences in gene expression across the transcriptome of the copepod Tigriopus californicus
}

Sean D Schoville ${ }^{1 *}$, Felipe S Barreto ${ }^{1}$, Gary W Moy ${ }^{1}$, Anastasia Wolff ${ }^{2}$ and Ronald S Burton ${ }^{1}$

\begin{abstract}
Background: Geographic variation in the thermal environment impacts a broad range of biochemical and physiological processes and can be a major selective force leading to local population adaptation. In the intertidal copepod Tigriopus californicus, populations along the coast of California show differences in thermal tolerance that are consistent with adaptation, i.e., southern populations withstand thermal stresses that are lethal to northern populations. To understand the genetic basis of these physiological differences, we use an RNA-seq approach to compare genome-wide patterns of gene expression in two populations known to differ in thermal tolerance.

Results: Observed differences in gene expression between the southern (San Diego) and the northern (Santa Cruz) populations included both the number of affected loci as well as the identity of these loci. However, the most pronounced differences concerned the amplitude of up-regulation of genes producing heat shock proteins (Hsps) and genes involved in ubiquitination and proteolysis. Among the hsp genes, orthologous pairs show markedly different thermal responses as the amplitude of hsp response was greatly elevated in the San Diego population, most notably in members of the $h s p 70$ gene family. There was no evidence of accelerated evolution at the sequence level for hsp genes. Among other sets of genes, cuticle genes were up-regulated in SD but down-regulated in SC, and mitochondrial genes were down-regulated in both populations.
\end{abstract}

Conclusions: Marked changes in gene expression were observed in response to acute sub-lethal thermal stress in the copepod T. californicus. Although some qualitative differences were observed between populations, the most pronounced differences involved the magnitude of induction of numerous hsp and ubiquitin genes. These differences in gene expression suggest that evolutionary divergence in the regulatory pathway(s) involved in acute temperature stress may offer at least a partial explanation of population differences in thermal tolerance observed in Tigriopus.

Keywords: Next-generation sequencing, RNA-seq, Heat shock proteins, Diversification, Temperature stress, Climate

\footnotetext{
* Correspondence: sschoville@ucsd.edu

'Marine Biology Research Division, Scripps Institution of Oceanography,

University of California, San Diego, 9500 Gilman Drive, La Jolla, CA

92093-0202, U.S.A

Full list of author information is available at the end of the article
} 


\section{Background}

Thermal environments often vary spatially and temporally across the geographic range of a species and such variation can be an important selective force leading to genetic divergence of local populations [1]. In particular, selection resulting from the extremes in local temperature variation can have a broad impact on an organism's genome, due to the important role of temperature on a range of biochemical and physiological processes [2]. Several studies have shown that tolerance of intertidal invertebrates to thermal stress is correlated with local temperature regimes; much of this work has focused on the tolerance limits of different species, both among unrelated taxa [3] and among congeners [4-6]. Results of these studies typically indicate that the geographic and ecological ranges of species are determined by thermal constraints, but the genetic basis for thermal tolerance is often not known and in some cases the observed phenotypic variation is driven by plasticity in traits rather than genetic differences among populations $[7,8]$. In order for local adaptation to evolve across environmental gradients, there must be underlying genetic variation in the phenotypic trait and moderate to strong environmental selection [9]. Additionally, rates of interpopulation gene flow must remain low, or gene swamping will override the effect of diversifying selection [10].

Several empirical studies have found evidence of the genetic fixation of alternative alleles related to local adaptation of populations along latitudinal clines [11,12] and altitudinal gradients [13,14]. These studies suggest that local adaptation sometimes involves structural changes in single protein-coding loci with large effects $[15,16]$. Point mutations in protein coding sequences can result in allelic gene products with differing thermal optima [17] and directly impact protein function (e.g., transporter affinity or enzyme activity) or alter the thermal stability of proteins, i.e., their propensity to lose native conformation at warmer temperatures. However, not all environmental adaptations involve changes in protein structure, as genes can alternatively evolve new patterns of expression. Natural selection on regulatory variation might play a major role in evolution of local adaptation $[18,19]$, particularly since a single change in a master regulator gene can result in widespread changes in gene expression across the transcriptome [20]. Of course, there is also the possibility that genetic changes underlying local adaptation involve a combination of both structural changes and changes in gene regulation. Efforts to differentiate these alternatives are only now becoming feasible with next generation sequencing (permitting the analysis of regulatory elements on a genome-wide scale) and studies of transcriptome responses to the environment.

Until relatively recently, assessing structural variation of genes and gene expression during thermal stress in non-model organisms has often been limited to few genes. Heat shock proteins (Hsps) have been the most important targets for investigation because they play an important role in maintaining cellular functions under environmental stress [4,21-26]. With the recent accessibility of genomic tools for use in non-model organisms, several studies have taken advantage of microarray technology for profiling expression of hundreds to thousands of genes simultaneously. These investigations have shown that the gene-regulatory response of intertidal animals to thermal stress is much more complex than previously predicted. For instance, Lockwood et al. [5] showed that different species of Mytilus mussels rely, at least partially, on different sets of genes (including Hsps) during heat shock experiments. Moreover, microarray studies have revealed classes of genes that were previously unmonitored during thermal stress, but that appear to play a significant role in the thermal response [5,27-29]. Next-generation RNA sequencing technology (RNA-seq) [30] now allows us to simultaneously assemble transcriptomes and quantify gene expression across tens of thousands of genes without any a priori genomic information. Another useful aspect of the RNA-seq approach is that the data consist of actual transcript sequences, permitting accurate determination of expression levels of closely related paralogs in gene-rich families (such as the Hsps) that might otherwise be pooled in microarray studies [31], and identification of any structural variation among copies of ortholgous genes found in different populations.

Here we initiate investigations of the genetic mechanisms that underlie population differences in thermal tolerance observed in Tigriopus californicus [32]. Besides experiencing extreme daily and seasonal temperature fluctuations (annual range of $6-33^{\circ} \mathrm{C}$ at one site) in their local shallow intertidal pools [33], this species is distributed over $5000 \mathrm{~km}$ of coastline, from Baja California, Mexico to Alaska, spanning 35 degrees of temperate latitude. Under moderate temperature conditions, populations of $T$. californicus show no consistent differences in survivorship [34]. Willett [32] and Kelly et al. [35] independently demonstrated that $T$. californicus exhibits pronounced interpopulation differences in tolerance to high temperature stress. With large local populations, restricted gene flow, and short generational time, we hypothesize that differences in high temperature tolerance are driven by local adaptation. As a first step in understanding the molecular mechanisms that might underlie adaptation to local thermal regimes among T. californicus populations, we employed RNAseq to characterize divergence in transcriptome-wide gene expression profiles between two populations with different thermal tolerances. We additionally examine the structural variation underlying members of the hsp70 gene family to examine whether sequence 
variability might account for differences between these populations.

\section{Methods}

\section{Preparation of animals}

At least 2000 Tigriopus californicus were collected from each of two natural populations, San Diego (SD: N 32.746842, W -117.254650) and Santa Cruz (SC: N 36.949589, W -122.047036), and maintained in the laboratory at $20^{\circ} \mathrm{C}$ with a 12-hour light:dark cycle for 1-3 generations before initiation of experiments. Two replicate samples of over 300 individual copepods of all life stages were pooled from laboratory stocks of SD and SC and flushed repeatedly in filtered ( 0.22 micron) seawater over two days. One sample from each population served as a control treatment, while the other sample served as the acute heat stress treatment. Pooling of large numbers of individuals into single RNA samples allows for biological averaging, and for small experimental designs, pooling has been shown to approximate non-pooled, replicated designs [36]. To initiate an acute heat stress, samples were placed in 50-ml Falcon tubes and immersed in a $35^{\circ} \mathrm{C}$ water bath for one hour, allowed to recover for 1 hour at $20^{\circ} \mathrm{C}$, and then immediately transferred into Tri Reagent (Sigma) for RNA extraction. Samples in the control treatment were maintained at $20^{\circ} \mathrm{C}$ and otherwise treated identically. Samples in Tri-Reagent were disrupted with a tissue homogenizer and total RNA was then extracted following the manufacturer's protocol.

\section{Illumina sequencing}

The Illumina mRNA sequencing kit (RS-100-0801) and multiplexing kit (PE-400-1001) were used to isolate mRNA and prepare sequencing libraries. Matching barcodes were assigned to the SD control and heat stress sample (TGACCA), as well as the SC control and heat stress samples (GCCAAT), to allow for multiplexing on two lanes of an Illumina GA-II genome analyzer flow cell. This was done to remove possible PCR bias in comparisons of control versus heat stress samples. For sequencing, the two barcoded control samples were pooled in one lane, while the two barcoded heat stress samples pooled in an adjacent lane of the flow cell. Previous experiments have suggested that there is relatively little between-lane bias [37]. Each sample was sequenced for single-end 100 basepair read lengths.

\section{Preparation of reference assemblies}

Reference transcriptomes for SD and SC were previously developed from 454 pyrosequencing of each population [38]. To improve coverage and representation of rare gene transcripts in these reference assemblies, we performed de novo assembly using the previously published 454 data and the new Illumina data. Separate assemblies were made for SD and SC sequencing reads. CLC Genomics Workbench v4.5 (CLC Bio) was used to remove adaptors and trim sequences of low quality reads, and the reads were assembled into contigs with the following assembly parameters: minimum fraction length of read overlap $=0.5$, minimum sequence similarity $=0.9$, minimum contig length 60 basepairs.

Assembled contigs from each population were identified as putative gene products using BLASTX searches in NCBI's non-redundant (nr) protein database and annotated for gene function using the Blast2GO software $[39,40]$. The highest scoring BLAST hit, at a threshold E-value of $10^{-3}$, was used to assign a gene name to each assembled contig. For contigs with a positive BLAST hit, Gene Ontology (GO) [41] terms were retrieved at a threshold of $10^{-6}$.

Orthology between SD and SC contigs was assessed by a reciprocal best-hit approach. We used NCBI's executable scripts to create BLAST-searchable databases with each set of contigs and then performed BLASTN searches between the two data sets. We parsed the output keeping only pairs of sequences that were each other's best hit, with $\mathrm{E} \leq 10^{-20}$, as putatively homologous.

\section{Read mapping and expression analysis}

Illumina reads from SD and SC samples were mapped to their respective reference assembly using CLC Genomics Workbench (read mapping parameters: minimum fraction length of read overlap $=0.5$, minimum sequence similarity $=0.99$ ). The expression of each gene was determined by calculating the sum of the reads mapping to each contig, and then normalized by dividing the number of observed reads by the length of the contig and by the total number of reads sequenced across the transcriptome (reads per kilobase per million reads, or RPKM). To compare levels of expression between control and heat stressed samples in each population, we used Kal's Z-test of proportions [42]. The $Z$-test calculates the difference in the proportion of reads observed between two treatments and compares whether each sample was drawn from the same distribution, using the normal distribution as an approximation of the binomial distribution. Due to the large number of tests, statistical significance was assessed using $p$-values adjusted by Bonferroni correction. In some cases Bonferroni can be overly conservative, so we consider tests as significant with a Bonferroni $p$-value $\leq 0.10$. This was more conservative than applying a false discovery rate at $p<0.05$, which detected many more $(>10 \mathrm{x})$ contigs. We then filtered the dataset of significant loci and retained only genes where at least one of the orthologous contigs had $>10$ mapped reads. For graphical purposes, RPKM values were $\log _{10}$ transformed due to the large range in expression values across genes. Using the GO terms for each transcriptome, we examined whether the group of genes 
showing a significant change in expression in each population were enriched for particular gene ontology terms relative to their reference transcriptome. Statistical significance was assessed using Fisher's exact tests implemented in the Blast2GO software.

\section{Evolutionary analysis of the Hsp70 family}

As discussed below, RNA-seq results identified 18 loci annotated as $h s p 70$. Because these genes were prominent in the genes responsive to thermal stress, we carried out a more detailed analysis of this gene family. We performed additional BLASTX against the Drosophila melanogaster and Caenorhabditis elegans genomes in order to identify T. californicus contigs that were paralogous $h s p 70$ loci. For each identified $h s p 70$ copy, we aligned the orthologs between SD and SC and performed comparative analyses of sequence divergence. To look for evidence of molecular adaptation, coding sequences were compared to calculate the ratio of non-synonymous $\left(d_{\mathrm{N}}\right)$ to synonymous $\left(d_{\mathrm{S}}\right)$ nucleotide substitution rates [43]. Typically, $d_{\mathrm{N}} / d_{\mathrm{S}}$ ratios much greater than one indicate strong diversifying selection, whereas values close to one are compatible with neutral evolution and values significantly less than one are consistent with balancing or purifying selection. To examine relationships among the Hsp70 proteins, orthologs of Drosophila melanogaster [GenBank: NM 169441.1, NM_079632.5, NM_079615.3, NM_080059.2, NM_169469.1, NM_080188.2, NM_141952.2, NM_167 306.1, NM_079017.2], Caenorhabditis elegans [EMBL: F26D10, C15H9, F43E2, C37H5, F44E5.4, F44E5.5, C12C8, F11F1], and Escherichia coli [UniProt: P77319] were obtained as out-group sequences and amino acids were aligned using the ClustalW2 algorithm [44]. A neighbor-joining tree was constructed in Mega5 [45], using the p-distances model with pairwise deletion of gaps and missing data. Support for topological relationships in the phylogram was evaluated by bootstrapping with 1,000 replicates.

\section{Validation of RNA-seq expression levels}

In order to evaluate the accuracy of the RNA-seq expression profiles, we measured the expression of a set of heat shock genes using quantitative PCR (qPCR). We first quantified expression using the same RNA samples prepared for RNA-seq, as a means of technical replication. cDNA from the transcriptome samples was diluted to $\sim 100 \mathrm{ng} / \mathrm{ul}$ and $1 \mathrm{ul}$ was amplified over 25 cycles with $200 \mathrm{nM}$ of each gene specific primer (Additional file 1) using Brilliant III Ultra-Fast SYBR Green QPCR Mastermix (Agilent Technologies). Several housekeeping genes were chosen to normalize the expression of target genes, including beta-actin, glyceraldehyde 3-phosphate dehydrogenase, tubulin, and myosin. Ct values for each sample were normalized by geometric averaging of the housekeeping genes, implemented in the program GeNorm [46].

We assessed biological accuracy further by replicating the thermal stress experiment with additional samples (biological replicates). For each replicate, thirty individuals were placed in $15-\mathrm{mL}$ tubes containing filtered seawater, and the experimental thermal stress was repeated as above. RNA was extracted using Tri-Reagent, with tissue homogenization performed using a Mini-BeadBeater 8 (BioSpec) with silica beads $(0.175 \mathrm{mg})$ at full speed for 30s. RNA was quantified by spectrophotometry, and 500 ng of RNA were used for cDNA synthesis with the High Capacity RNA-to-cDNA kit (Applied Biosystems). The experiment was performed with three replicates per temperature for each population. Quantitative PCR was performed in $20-\mu \mathrm{L}$ reactions with the iTaq Fast SYBR Green Supermix (Bio-Rad), and contained $2 \mu \mathrm{L}$ of a 1:10 dilution of cDNA and $250 \mathrm{nM}$ of each primer. Fluorescence was measured in a Stratagene Mx 3000P System (Stratagene), with the following thermal profile: $95^{\circ} \mathrm{C}$ for $3 \mathrm{~min}$, followed by 40 cycles of $95^{\circ} \mathrm{C}$ for $10 \mathrm{~s}$ and $59^{\circ} \mathrm{C}$ for $30 \mathrm{~s}$. At the end of thermal cycling, a melting curve analysis was performed to confirm the presence of single amplification products. A total of 14 target genes were surveyed, and their expression levels were normalized to the expression of housekeeping genes.

\section{Results}

Illumina sequencing, de novo assembly, and RNA-seq mapping

The sequencing data from this study have been submitted to the NCBI Gene Expression Omnibus under accession no. GSE38546. Single-end 100 basepair Illumina sequencing of the SD samples resulted in 9,292,573 reads, with $6,794,534$ reads in the heat stress sample and 2,498,039 reads in the control sample. Sequencing of the SC samples yielded 10,794,996 reads, with 6,205,028 reads in the heat stress sample and 3,256,758 in the control sample. We combined samples from each population with previous 454 sequencing data [38] and assembled the reads into 66,943 unique SD contigs and 60,648 unique SC contigs. The average length of the SD contigs was 498 basepairs (range: 49-9,830 bp) and the average length of the SC contigs was 520 bp (range: 36-9,003 bp). These contigs formed the reference transcriptomes used in our RNA-seq gene expression analysis. Approximately $29 \%$ and 33\% of the SD and SC assemblies, respectively, were annotated using BLAST, and a total of 19,622 contigs were identified as orthologs based on reciprocal BLAST to each transcriptome. Approximately $78 \%$ of the total reads were uniquely mapped in the SD control sample, while $\sim 82 \%$ reads were uniquely mapped in the SD heat stress sample. In the SC control sample, $\sim 71 \%$ reads were uniquely mapped, while $\sim 80 \%$ reads were uniquely mapped in the SC heat stress 
sample. A distribution of the RPKM values shows that most of the contigs in each treatment had values around 10 RPKM, with slightly lower values in the heat stress samples (see Additional file 2).

\section{Differential gene expression}

In the comparison of SD control and heat shock samples, a total of 590 contigs $(0.88 \%$ of 66,943 contigs) showed evidence of a statistically significant difference in expression (Bonferroni corrected $p<0.10$ ). Of these 590 contigs, 234 were removed due to a low number of mapped reads, leaving $356(0.53 \%)$ as significantly up or down regulated. In the comparison of SC control and heat shock samples, a total of 903 contigs $(1.49 \%$ of 60,648 contigs) showed evidence of a statistically significant difference in expression (Bonferroni corrected $p<0.10$ ). Of these 903 contigs, 36 were removed due to a low number of mapped reads, leaving $867(1.43 \%)$ as significantly up or down regulated. Most of the contigs identified as significant had RPKM values between 10 and 100, with better coverage than the overall transcriptome (see Additional file 2).
We examined the functional classes of genes that showed the significant changes in expression and tested for enrichment of gene ontology terms relative to their frequency in each transcriptome. Among the gene ontology terms that were significantly enriched (Table 1), genes responding to stress and genes involved in cuticle formation were the most overrepresented in SD and ribosomal genes and lipid transport genes were the most overrepresented in the SC population. We also focused on the pattern of expression in four classes of genes that showed significant fold changes in both populations: heat shock proteins, genes associated with proteolysis, mitochondrial genes, and genes associated with cuticle formation (see Additional file 3 for frequency of other gene ontology terms). We plotted the log-transformed expression of genes that were significantly up or down-regulated in the heatshock treatment relative to control samples (Figure 1). Plots of the SD heat stress versus control sample (Figure 1A) show that variation in up-regulated gene expression was greater than in down-regulated genes, with a group of 125 genes exhibiting a $>10 \mathrm{X}$-fold up-regulation relative to

Table 1 Enrichment of gene ontology (GO) terms in genes that were differentially expressed during heat stress

\begin{tabular}{|c|c|c|c|c|}
\hline \multicolumn{5}{|c|}{ Enriched in SD } \\
\hline \multirow[t]{2}{*}{ GO-ID } & \multirow[t]{2}{*}{ Category: Term } & \multicolumn{2}{|c|}{ \% sequences within group* } & \multirow[t]{2}{*}{ FDR $P$-value } \\
\hline & & differentially expressed & non-changing & \\
\hline 0006950 & $\mathrm{P}$ : response to stress & 24.07 & 2.47 & $3.94 \times 10^{-5}$ \\
\hline 0042302 & F: structural constituent of cuticle & 9.84 & 0.19 & $3.94 \times 10^{-5}$ \\
\hline 0008199 & F: ferric iron binding & 4.69 & 0.01 & 0.00592 \\
\hline 0006826 & P: iron ion transport & 4.69 & 0.03 & 0.00630 \\
\hline 0006879 & P: cellular iron ion homeostasis & 4.69 & 0.03 & 0.00630 \\
\hline 0005524 & F: ATP binding & 31.37 & 7.52 & 0.00880 \\
\hline 0051093 & P: negative regulation of developmental process & 8.06 & 0.48 & 0.01300 \\
\hline \multicolumn{5}{|c|}{ Enriched in SC } \\
\hline \multirow[t]{2}{*}{ GO-ID } & \multirow[t]{2}{*}{ Category: Term } & \multicolumn{2}{|c|}{$\%$ sequences within group ${ }^{\#}$} & \multirow[t]{2}{*}{ FDR $P$-valu } \\
\hline & & differentially expressed & non-changing & \\
\hline 0005319 & F: lipid transporter activity & 4.46 & 0.11 & $1.27 \times 10^{-8}$ \\
\hline 0006869 & P: lipid transport & 4.46 & 0.23 & $1.50 \times 10^{-6}$ \\
\hline 0033279 & C: ribosomal subunit & 6.36 & 1.24 & $7.46 \times 10^{-4}$ \\
\hline 0032982 & C: myosin filament & 2.63 & 0.25 & 0.01390 \\
\hline 0008199 & F: ferric iron binding & 1.74 & 0.07 & 0.01914 \\
\hline 0006879 & P: cellular iron ion homeostasis & 1.74 & 0.08 & 0.02918 \\
\hline 0006414 & P: translational elongation & 3.54 & 0.62 & 0.03517 \\
\hline 0003677 & F: DNA binding & 0.86 & 6.18 & 0.03977 \\
\hline 0006826 & P: iron ion transport & 1.74 & 0.10 & 0.03977 \\
\hline 0009001 & F: serine O-acetyltransferase activity & 1.30 & 0.03 & 0.04109 \\
\hline 0031224 & C: intrinsic to membrane & 3.08 & 10.22 & 0.04240 \\
\hline
\end{tabular}

Bold and italic fonts indicate terms that were over- or under-represented, respectively, in the differentially expressed category, based on false discovery rate (FDR)corrected Fisher's exact tests. $\mathrm{GO}$ categories: $\mathrm{F}=$ function, $\mathrm{P}=$ process, $\mathrm{C}=$ cellular component.

*Based on number of annotated genes in SD: differentially expressed - 67; non-changing - 6980 .

\#Based on number of annotated genes in SC: differentially expressed - 234; non-changing - 7289. 

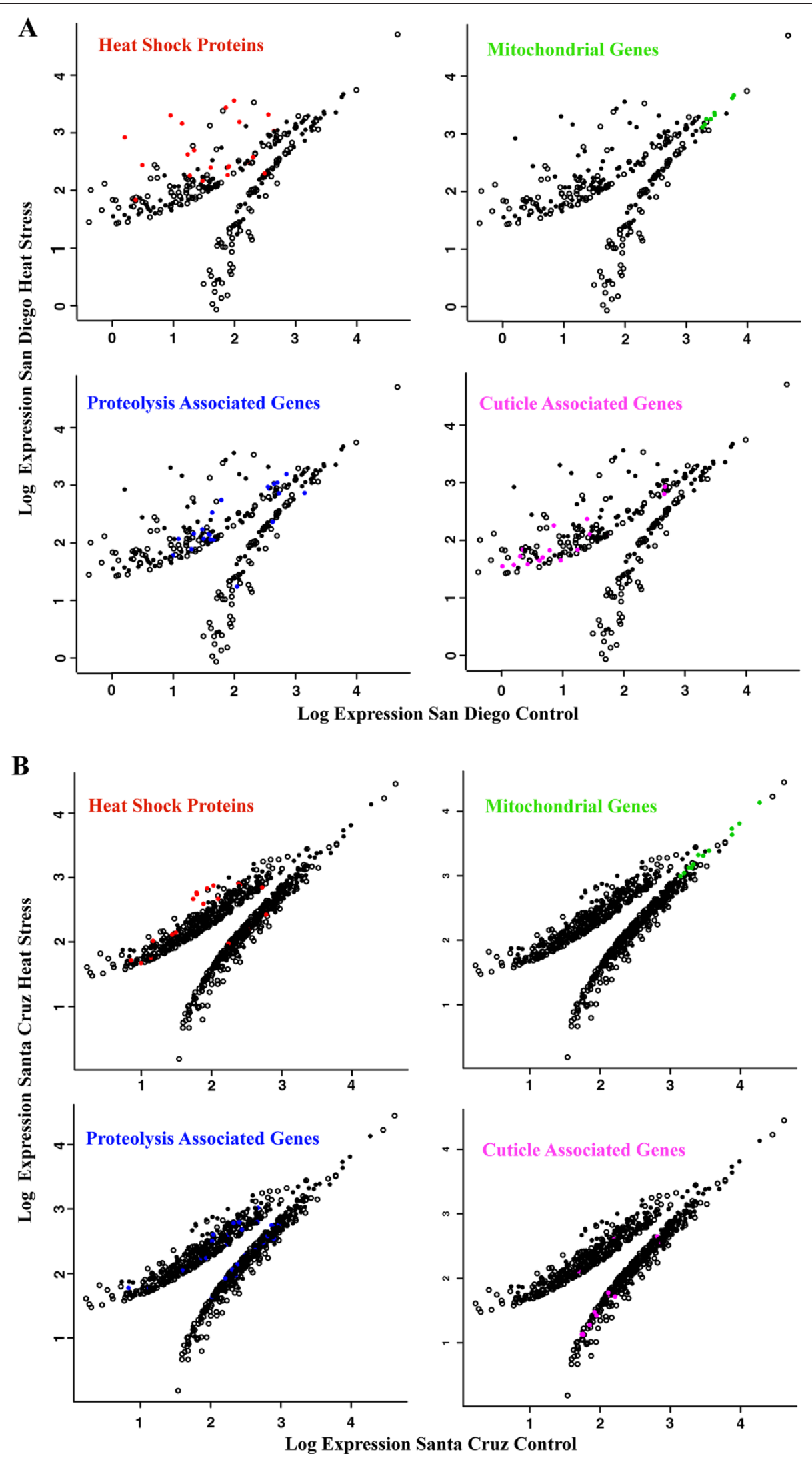

Figure 1 (See legend on next page.) 
the control sample. Thirteen genes with $>10 \mathrm{X}$-fold upregulation were identified as heat shock proteins (Hsps). Nineteen genes involved in the degradation of damaged proteins (proteolysis associated genes) were up-regulated, as were 20 genes involved in cuticle formation. Downregulated genes include eight mtDNA-encoded genes involved in cellular respiration, as well as some other classes of genes (e.g. lipid transport and hydrolases, not shown). In comparison, plots of the SC heat stress versus control sample (Figure 1B) show relatively even distribution of up-regulated and down-regulated genes, with much less variation in expression than the SD treatment. Only 15 genes show a $>10 \mathrm{X}$-fold up-regulation, and two of these are hsps. Seventeen proteolytic genes were up-regulated and eighteen were down-regulated in the SC comparison. Thirteen mitochondrial genes in SC were down-regulated and show roughly the same response as in SD. In striking contrast to the SD comparison, thirteen SC genes involved in cuticle formation were largely down-regulated.

Although the direction of change in expression under heat stress is the same in both populations across most genes (with cuticle-associated genes being an exception), the magnitude of up- or down-regulation is markedly different. In the SD sample, several genes showed $>100$ fold change in expression, while the greatest change in the SC sample was 25-fold (Table 2; Figure 1). An examination of the identity of genes with statistically significant changes in expression revealed that 63 were shared in both populations (Table 2). Twenty-five of these were up-regulated in both populations, including eight heat shock proteins. In all cases, the heat shock proteins showed higher fold changes in the SD population. Fourteen genes were down-regulated in both populations, including 7 mitochondrial genes with very similar levels of change in both populations. Finally, 24 genes showed population-specific direction of responses, 12 of which belonged to two functional classes showing opposite expression profiles. Seven cuticle proteins were up-regulated in SD (mean $=16.1$-fold) and down-regulated in SC (-3.1-fold), while 5 hydrolases were down-regulated in SD (-2.9-fold) and up-regulated in SC (2.4-fold) (Table 2).

When comparing transcriptional profiles of hsps, some population-specific responses to heat shock were also observed (Table 3). A total of 29 putatively unique $h s p$ transcripts were identified as orthologs, only nine of which showed significant change in the same direction (up-regulation) in both samples. For each of $10 \mathrm{SC}$ hsp70 paralogs that can be matched to an SD ortholog, the patterns of induction during thermal stress are similar in the two populations. However, the amplitude of the stress response is markedly different, with the SD orthologs consistently showing a higher level of stress induction in the more thermally tolerant SD population. This pattern holds across other hsp genes, with $h s p$ induction invariably higher in SD. For 13 putative hsps, an orthologous copy was not identified in one population through reciprocal BLAST. These genes, missing from one transcriptome, most likely reflect an incomplete transcriptome assembly; alternatively a gene may have been lost in one of the populations. These hypotheses not yet been tested via PCR amplification from genomic DNA.

\section{Sequence divergence in the hsp70 gene family}

Average sequence divergence of the 29 orthologous hsp 70 pairs is $2.9 \%$ and ranges from $0.0-8.7 \%$ (Table 3 ). The ratio of rates of non-synonymous to synonymous substitutions $\left(d_{\mathrm{N}} / d_{\mathrm{S}}\right)$ is less than one in all pairs, providing no evidence for divergent selection. Based on full-length transcript sequences, we unambiguously identified eight hsp70 paralogs in the T. californicus transcriptome. We examined the relationship between these paralogs and Hsp70 proteins from Drosophila melanogaster and Caenorhabditis elegans. A neighbor-joining tree based on protein alignment (Figure 2) shows that five pairs have an ancient origin among Metazoans, while 3 pairs have diversified from an ancestral copy of Hsp70 \#2. In all cases, orthologous copies in SD and SC are more closely related to one another than to other Hsp70 paralogs. Notably, the relatively closely related group of paralogs (\#1, \#2, \#5, and \#16) show markedly different response to thermal stress.

\section{Validation of RNA-seq gene expression levels}

We performed qPCR assays on a subset of genes using the same RNA samples prepared for RNA-seq (a type of technical validation), as well as using RNA from additional samples (for biological validation). In nearly all comparisons (75\%), fold-changes in expression measured by qPCR were in the same direction as those estimated by RNA-seq (Table 4). Moreover, the magnitude of these changes were also highly congruent between the two methods, as tested by linear regressions of $\log _{2}$ folddifferences (technical replicates: $r=0.85, \quad F_{1,34}=87.4$, $P<0.001$; biological replicates: $r=0.84, \quad F_{1,26}=68.2$, $P<0.001)$. 


\begin{tabular}{|c|c|c|c|c|c|}
\hline \multirow[b]{2}{*}{ Contig identification } & \multicolumn{2}{|c|}{$\begin{array}{l}\text { Fold } \\
\text { change }\end{array}$} & \multirow[b]{2}{*}{ Gene Ontology annotation } & \multirow[b]{2}{*}{$\begin{array}{c}\text { SD } \\
\text { Accession }\end{array}$} & \multirow[b]{2}{*}{$\begin{array}{c}\text { SC } \\
\text { Accession }\end{array}$} \\
\hline & SD & SC & & & \\
\hline \multicolumn{6}{|l|}{ Up-regulated in both } \\
\hline hspbeta-1 \#2 & 22.9 & 4.5 & response to stress; response to heat & JW506232 & JW525782 \\
\hline hspbeta-1 \#3 & 24.6 & 4.2 & response to stress & JW506234 & JW525781 \\
\hline hspbeta-1 \#4 & 105.2 & 4.7 & response to heat & JW506233 & JW525780 \\
\hline hsp20 \#1 & 38.17 & 3.0 & response to stress; response to heat & JW519178 & JW525783 \\
\hline hsp70 \#1 & 2.5 & 1.33 & ATP binding; response to stress; auxin biosynthetic process & JW519142 & JW525772 \\
\hline hsp70 \#2 & 224.3 & 7.86 & $\begin{array}{l}\text { mitotic cell cycle; auxin biosynthetic process; response to heat; ATP binding; response to xenobiotic stimulus; } \\
\text { DNA replication initiation; camera-type eye morphogenesis; response to cadmium ion; negative regulation of apoptosis; } \\
\text { nucleus }\end{array}$ & JW519350 & JW526654 \\
\hline hsp70 \#5 & 13.0 & 3.8 & ATP binding; response to stress; auxin biosynthetic process & JW506905 & JW526653 \\
\hline hsp70 \#9 & 460.3 & 7.3 & ATP binding; response to stress; auxin biosynthetic process & N/A & N/A \\
\hline hsp90 \#1 & 5.8 & 3.3 & protein folding; cytoplasm; unfolded protein binding; ATP binding; response to stress & JW506231 & JW525777 \\
\hline Hypothetical protein & 1.3 & 1.4 & & JW506500 & JW533424 \\
\hline Involucrin, putative & 12.6 & 3.8 & apoptosis; protein binding & JW507027 & JW526798 \\
\hline $\begin{array}{l}\text { Map kinase-interacting serine } \\
\text { Threonine-protein kinase } 1\end{array}$ & 1.7 & 2.3 & & JW507795 & JW527737 \\
\hline Midline fasciclin & 3.6 & 4.9 & & JW508028 & JW527995 \\
\hline Polyubiquitin & 2.2 & 2.5 & viral protein processing; proteolysis; serine-type endopeptidase activity; EC 3.4.21.0 & JW519561 & JW535653 \\
\hline Transcription factor kayak & 3.6 & 4.3 & $\begin{array}{l}\text { regulation of transcription, DNA-dependent; sequence- specific DNA binding; protein dimerization activity; DNA binding; } \\
\text { transcription factor activity; nucleus }\end{array}$ & JW519889 & JW538428 \\
\hline Ubiquitin & 2.4 & 2.4 & & JW519952 & JW532240 \\
\hline Unknown & 1.1 & 2.1 & & JW513666 & JW533072 \\
\hline Unknown & 7.1 & 3.7 & & JW513718 & JW529892 \\
\hline Unknown & 174.3 & 25.3 & & JW514975 & JW531801 \\
\hline Unknown & 46.2 & 9.4 & & N/A & N/A \\
\hline Unknown & 1.8 & 1.4 & & N/A & $\mathrm{N} / \mathrm{A}$ \\
\hline Unknown & 16.4 & 1.9 & & N/A & \\
\hline Unknown & 4.3 & 2 & & JW518153 & $\mathrm{N} / \mathrm{A}$ \\
\hline Unknown & 6.2 & 1.8 & & JW518470 & JW532411 \\
\hline Unknown & 460.3 & 7.3 & & N/A & N/A \\
\hline
\end{tabular}


Table 2 Genes found in both populations with statistically significant changes in expression after thermal stress (Continued)

Down-regulated in both

\section{0 s ribosomal protein L14}

ATP synthase subunit 6

Cellular retinoic acid- binding

protein 2

Cytochrome oxidase subunit 3

Ferritin heavy chain 1

Ferritin middle subunit 216/heavy chain polypeptide 1

$\mathrm{NADH}$ dehydrogenase $4 \mathrm{~L}$

NADH dehydrogenase subunit 1

NADH dehydrogenase subunit 2

NADH dehydrogenase subunit 4

$\mathrm{NADH}$ dehydrogenase subunit 5

$\mathrm{NADH}$ dehydrogenase subunit 6

Nuclear protein 1/Serine threonineprotein phosphatase

\section{Unknown}

\section{Population-specific direction of response}

3,4-dihydroxy-2- butanone-4phosphate synthase

Cell wall-associated hydrolase

Cell wall-associated hydrolase

Cell wall-associated hydrolase

Cell wall-associated hydrolase

Cell wall-associated hydrolase

Conserved protein

Conserved protein

Cuticle protein 248

Cuticle protein 281/502

Cuticle protein 283/409

Cuticle protein 541/577

Cuticle protein 645/704

Cuticle protein $7 / 414$
0.790 .58 intracellular

0.780 .67 oxidation reduction; mitochondrial

0.610 .49 lipid binding; binding; transporter activity; transport

0.8160 .69 oxidation reduction; mitochondrial

0.480 .49 transition metal ion binding; cellular iron ion homeostasis; oxidoreductase activity; ferric iron binding; oxidation reduction; iron ion transport

0.710 .61 cellular iron ion homeostasis; oxidoreductase activity; ferric iron binding; oxidation reduction; iron ion transport

0.730 .67 oxidation reduction; mitochondrial

0.710 .7 oxidation reduction; mitochondrial

0.740 .69 oxidation reduction; mitochondrial

0.730 .71 oxidation reduction; mitochondrial

0.850 .73 oxidation reduction; mitochondrial

0.710 .67 oxidation reduction; mitochondrial

$0.67 \quad 0.56$

0.42 2.81 metal ion binding; riboflavin biosynthetic process; isomerase activity; EC 4.1.99.12

0.641 .67 hydrolase activity

$0.53 \quad 3.39$ hydrolase activity

0.392 .41 hydrolase activity

$0.23 \quad 1.6$ hydrolase activity

$0.23 \quad 2.9$ hydrolase activity

$0.61 \quad 2.9$

$0.57 \quad 3.0$

32.90 .46 structural constituent of cuticle

2.30 .3 structural constituent of cuticle

36.60 .24 structural constituent of cuticle

$11.0 \quad 0.27$ structural constituent of cuticle

9.30 .42 structural constituent of cuticle

4.6 0.32 structural constituent of cuticle
JW518618 JW521020

DQ913891 DQ917374

JW503430 JW522628

DQ913891 DQ917374

JW505400 JW524830

N/A JW524829

DQ913891 DQ917374

DQ913891 DQ917374

DQ913891 DQ917374

DQ913891 DQ917374

DQ913891 DQ917374

DQ913891 DQ917374

JW508743 JW537408

JW517373 JW531354

JW517629 N/A

JW503411 JW522621

JW507653 JW527565

JW503414 JW527566

JW503409 JW522614

N/A JW522624

JW505232 JW524634

JW504216 JW523492

JW504378 JW523655

JW504361 JW523649

JW504364 JW523634

JW504368 JW523638

JW504358 JW523633

JW504393 JW523650 
Table 2 Genes found in both populations with statistically significant changes in expression after thermal stress (Continued)

\begin{tabular}{|c|c|c|c|c|c|}
\hline Ferritin chloroplast expressed & 2.9 & 0.61 & iron ion homeostasis; oxidoreductase activity; iron binding & JW519009 & JW524836 \\
\hline Elegans protein & 0.61 & 2.0 & $\begin{array}{l}\text { signal transducer activity; membrane; signal transduction; embryonic development ending in seed dormancy; } \\
\text { protein binding; cellular component }\end{array}$ & JW505050 & JW524418 \\
\hline Hypothetical protein & 1.5 & 0.65 & & JW519182 & JW526134 \\
\hline Hypothetical protein & 0.44 & 3.7 & & JW506539 & JW526192 \\
\hline Hypothetical protein & 0.24 & 4.1 & & JW506786 & JW526524 \\
\hline Leucine-rich protein & 0.46 & 4.6 & & JW519332 & JW526187 \\
\hline Serine acetyltransferase & 0.69 & 3.1 & acyltransferase activity & JW504201 & JW537250 \\
\hline Unknown & 4.3 & 0.19 & & N/A & N/A \\
\hline Unknown & 2.1 & 0.69 & & JW518261 & JW532321 \\
\hline Unknown & 2.0 & 0.47 & & JW517851 & JW533144 \\
\hline Unknown & 1.7 & 0.69 & & JW518175 & JW525297 \\
\hline
\end{tabular}

$\mathrm{N} / \mathrm{A}=$ contigs for which a NCBI accession was not created because their sequences were shorter than the minimum length allowed in a Transcriptome Shotgun Assembly (TSA) submission. 
Table 3 RNA-seq quantification of gene expression in heat shock proteins

\begin{tabular}{|c|c|c|c|c|c|c|}
\hline \multirow[t]{2}{*}{ Contig Idenitification } & \multicolumn{2}{|c|}{ Fold change } & \multirow{2}{*}{$\begin{array}{c}\% \\
\text { Divergence }\end{array}$} & \multirow[t]{2}{*}{$d_{\mathrm{N}} / d_{\mathrm{S}}$ Ratio } & \multirow{2}{*}{$\begin{array}{c}\text { SD } \\
\text { Accession }\end{array}$} & \multirow{2}{*}{$\begin{array}{c}\text { SC } \\
\text { Accession }\end{array}$} \\
\hline & SD & SC & & & & \\
\hline hsf & 0.7 & 2.4 & 1.2 & 0.104 & JW505571 & JW525030 \\
\hline hspbeta-1 \#1 & 1.0 & 0.74 & 2.8 & 0.163 & JW504867 & JW522977 \\
\hline hspbeta-1 \#2 & $22.9^{*}$ & $4.5^{*}$ & 3.1 & 0.062 & JW506232 & JW525782 \\
\hline hspbeta-1 \#3 & $24.6^{*}$ & $4.2^{*}$ & 2.8 & 0.262 & JW506234 & JW525781 \\
\hline hspbeta-1 \#4 & $105.2^{*}$ & $4.7^{*}$ & 5.6 & 0.3 & JW506233 & JW525780 \\
\hline hspbeta-1 \#5 & $4.9^{*}$ & 2.1 & 2.3 & 0.085 & JW519143 & JW525778 \\
\hline hsp10 & 0.97 & $0.44^{*}$ & 3.2 & 0.035 & JW501904 & JW520834 \\
\hline hsp16 & $9.8^{*}$ & 1.3 & 4 & 0.349 & JW506236 & JW525786 \\
\hline hsp20 \#1 & $38.2^{*}$ & $3.0^{*}$ & 3.3 & 0.023 & JW519178 & JW525783 \\
\hline hsp20 \#2 & $\mathrm{n} / \mathrm{o}$ & $4.8^{*}$ & - & - & $n / o$ & N/A \\
\hline hsp40 \#1 & $1.9^{*}$ & 1.4 & 1.7 & 0 & JW506223 & JW525767 \\
\hline hsp40 \#2 & $3.2^{*}$ & 1.8 & 3 & 0.032 & JW506406 & JW526009 \\
\hline hsp60 & 1.1 & 0.74 & 2.5 & 0 & JW519415 & JW521007 \\
\hline hsp67b2 \#1 & 1.2 & $0.59^{*}$ & 8.7 & 0.029 & JW506224 & JW525770 \\
\hline hsp67b2 \#2 & 0.88 & $0.46^{*}$ & 2 & 0.222 & JW519141 & JW525771 \\
\hline hsc70 & $1.9^{*}$ & 1.6 & 1.2 & 0 & JW506426 & JW525790 \\
\hline hsp70 \#1 & $2.5^{*}$ & $1.3^{*}$ & 2.9 & 0.014 & JW519142 & JW525772 \\
\hline hsp70 \#2 & $224.3^{*}$ & $7.9^{*}$ & 3.9 & 0.035 & JW519350 & JW526654 \\
\hline hsp70 \#3 & 0.74 & 1.8 & 1.6 & 0 & JW506218 & JW525763 \\
\hline hsp70 \#4 & 0.92 & 1.3 & 1.9 & 0 & JW506217 & JW525759 \\
\hline hsp70 \#5 & $13.0^{*}$ & $3.8^{*}$ & 2.6 & 0.015 & JW506905 & JW526653 \\
\hline hsp70 \#6 & $218.9^{*}$ & $n / o$ & - & - & JW506227 & $\mathrm{n} / \mathrm{o}$ \\
\hline hsp70 \#7 & $28.2^{*}$ & $n / o$ & - & - & JW506228 & $n / o$ \\
\hline hsp70 \#8 & $524.4^{*}$ & $\mathrm{n} / \mathrm{o}$ & - & - & JW506906 & $\mathrm{n} / \mathrm{o}$ \\
\hline hsp70 \#9 & $460.3^{*}$ & $7.3^{*}$ & 3.7 & 0 & N/A & N/A \\
\hline hsp70 \#10 & $\mathrm{n} / \mathrm{o}$ & $8.5^{*}$ & - & - & $\mathrm{n} / \mathrm{o}$ & $\mathrm{N} / \mathrm{A}$ \\
\hline hsp70 \#11 & $n / o$ & $7.3^{*}$ & - & - & $n / o$ & $\mathrm{~N} / \mathrm{A}$ \\
\hline hsp70 \#12 & $\mathrm{n} / \mathrm{o}$ & $5.1^{*}$ & - & - & $n / o$ & JW525760 \\
\hline hsp70 \#13 & $\mathrm{n} / \mathrm{o}$ & $9.6^{*}$ & - & - & $\mathrm{n} / \mathrm{o}$ & JW525773 \\
\hline hsp70 \#14 & $\mathrm{n} / \mathrm{o}$ & $8.9^{*}$ & - & - & $\mathrm{n} / \mathrm{o}$ & JW525774 \\
\hline hsp70 \#15 & $6.1^{*}$ & 2.6 & 0 & 0.021 & JW502097 & JW521081 \\
\hline hsp70 \#16 & 2.7 & 1.3 & 3 & 0.043 & JW506226 & JW526652 \\
\hline
\end{tabular}


Table 3 RNA-seq quantification of gene expression in heat shock proteins (Continued)

\begin{tabular}{|c|c|c|c|c|c|c|}
\hline hsp70 \#17 & 0.99 & 1.8 & 4.2 & 0.061 & JW506216 & JW525758 \\
\hline hsp70 \#18 & 0.92 & 1.3 & 1.8 & 0 & JW506217 & JW525759 \\
\hline hsp70 interacting protein & $41.6^{*}$ & $n / o$ & - & - & $\mathrm{N} / \mathrm{A}$ & $n / o$ \\
\hline hsp70/90 organizing protein & $3.3^{*}$ & 0.83 & 2 & 0.023 & JW506431 & JW528118 \\
\hline hsp71 \#1 & $88.7^{*}$ & 3.6 & 4.4 & 0 & JW506229 & JW533019 \\
\hline hsp71 \#2 & $n / o$ & $7.1^{*}$ & - & - & $n / o$ & JW525776 \\
\hline hsp90 \#1 & $5.8^{*}$ & $3.3^{*}$ & 3.3 & 0.015 & JW506231 & JW525777 \\
\hline hsp90 \#2 & $n / o$ & $7.1^{*}$ & - & - & $n / o$ & N/A \\
\hline hsp105 & 1.9 & 1.6 & 1.1 & 0 & JW506426 & JW525790 \\
\hline mtDNA hsp & $37.0^{*}$ & $n / o$ & - & - & JW502693 & $\mathrm{n} / \mathrm{o}$ \\
\hline
\end{tabular}

Statistically significant changes indicated by an asterisk. Uncorrected percent sequence divergence and the non-synonymous $\left(d_{\mathrm{N}}\right)$ to synonymous $\left(d_{\mathrm{s}}\right)$ substitution ratio are shown for trimmed alignments. Gaps in the data due to missing orthologs are indicated as " $\mathrm{n} / \mathrm{o}$ ".

$\mathrm{N} / \mathrm{A}=$ contigs for which a NCBI accession was not created because their sequences were shorter than the minimum length allowed in a Trascriptome Shotgun Assembly (TSA) submission 


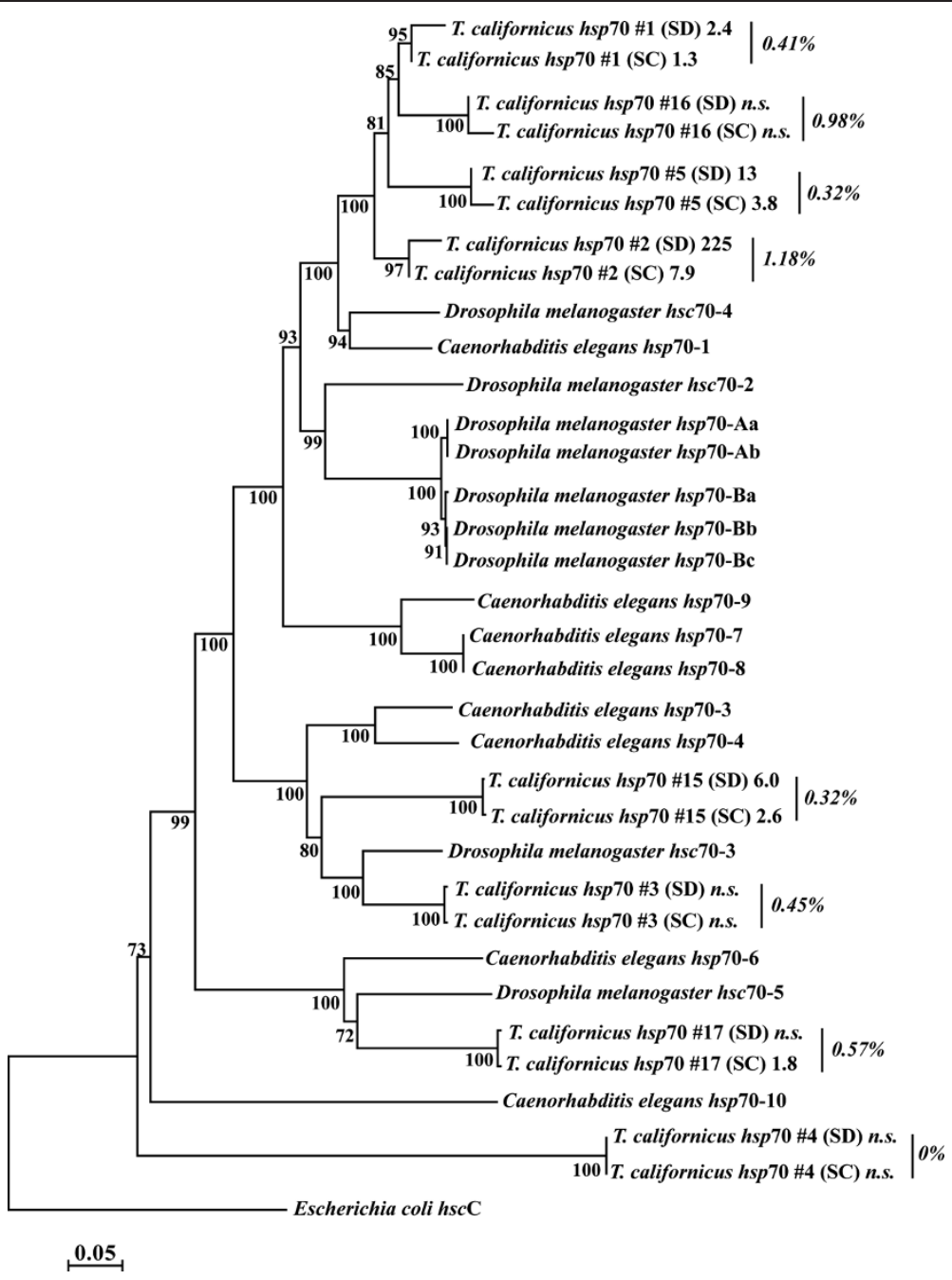

Figure 2 Neighbor-joining phylogram of Hsp70 proteins from Tigriopus californicus and out-groups. Numbers next to internal nodes represent bootstrap values from 1,000 replicates. For T. californicus, numbers next to population labels show the fold-change during thermal stress ('n.s.' = not significantly up- or down-regulated), as quantified by RNA-seq, while numbers associated with each pair of orthologs show the percent divergence in amino acid sequence between populations. NCBI accession numbers for T. californicus Hsp sequences can be found in Table 3.

\section{Discussion}

\section{Population divergence in acute heat stress}

Populations of Tigriopus californicus show strikingly different survivorship following acute thermal stress [32], with populations in southern California (i.e. San Diego) exhibiting higher survivorship. Here we tested the hypothesis that differences in patterns of gene expression play a role in adaptation to thermal stress. Our results, based on an RNA-seq approach, demonstrated that the two populations under study have genetically diverged in their transcriptome response to acute thermal stress. While the number of reads obtained for each treatment in each population limited our power to detect rare transcripts and incomplete assemblies could result in uneven representations of genes in each population, our qPCR data confirmed that the RNAseq results are robust measurements of gene expression changes in the tested populations. Our experiment showed that statistically significant changes in expression involved a small number of genes across the transcriptome of T. californicus (356 in San Diego and 867 in Santa Cruz, accounting for less than $1.5 \%$ of their respective assemblies). Among the subset of genes with statistically significant changes in expression, only 63 were responsive in both populations, suggesting that the two populations have diverged in the identity of affected loci. Furthermore, population differences were evident in both the direction and magnitude of expression changes in these 63 genes. The functional groups represented by these genes also differed between the two populations, as different gene ontology terms were enriched relative to the transcriptome. The San Diego population from 
Table 4 Comparison of RNA-seq and qPCR measurements of fold change in gene expression in selected genes

\begin{tabular}{|c|c|c|c|c|c|c|c|}
\hline & \multirow[t]{2}{*}{ Gene } & \multicolumn{2}{|c|}{ SD fold change } & \multicolumn{2}{|c|}{ SC fold change } & \multirow[t]{2}{*}{ SD accession } & \multirow[t]{2}{*}{ SC accession } \\
\hline & & RNA-seq & qPCR & RNA-seq & qPCR & & \\
\hline \multirow[t]{18}{*}{ Technical replicates } & Ubiquitin & 0.9 & 0.1 & 0.6 & 0.1 & JW512238 & JW538961 \\
\hline & hspbeta-1 \#2 & 22.9 & 20.6 & 4.5 & 2.1 & JW506232 & JW525782 \\
\hline & hspbeta-1 \#3 & 24.6 & 13.6 & 4.2 & 4.7 & JW506234 & JW525781 \\
\hline & hspbeta-1 \#4 & 105.2 & 109.5 & 4.7 & 2.3 & JW506233 & JW525780 \\
\hline & hspbeta-1 \#5 & 4.9 & 2.8 & 2.1 & 1.8 & JW519143 & JW525778 \\
\hline & hsp20 \#1 & 38.2 & 47 & 3 & 7.1 & JW519178 & JW525783 \\
\hline & hsp70 \#2 & 224 & 35.9 & 7.8 & 2.4 & JW519350 & JW526654 \\
\hline & hsp70 \#3 & 0.7 & 1.1 & 1.8 & 4.5 & JW506218 & JW525763 \\
\hline & hsp70 \#4 & 0.9 & 0.7 & 1.3 & 1.9 & JW506217 & JW525759 \\
\hline & hsp70 \#5 & 13 & 8.2 & 3.8 & 4.2 & JW506905 & JW526653 \\
\hline & hsp70 \#15 & 6.1 & 4.4 & 2.6 & 2.8 & JW502097 & JW521081 \\
\hline & hsp70 \#16 & 2.7 & 1.5 & 1.3 & 1.5 & JW506226 & JW526652 \\
\hline & hsp70 \#17 & 1 & 0.7 & 1.8 & 2.2 & JW506216 & JW525758 \\
\hline & DNAJ \#1 & 1.1 & 0.5 & 2.1 & 2.5 & JW503763 & JW522979 \\
\hline & DNAJ \#2 & 1.2 & 1.4 & 1.2 & 2 & JW504839 & JW524198 \\
\hline & DNAJ \#3 & 1.2 & 0.7 & 1 & 4.2 & JW503761 & JW522978 \\
\hline & DNAJ \#4 & 1.1 & 0.6 & 0.5 & 0.8 & JW503762 & JW522980 \\
\hline & hsp90 \#1 & 5.8 & 1.3 & 3.3 & 7.5 & JW506231 & JW525777 \\
\hline \multirow[t]{14}{*}{ Biological replicates } & hspbeta-1 \#3 & 24.6 & 30.7 & 4.2 & 7.3 & JW506234 & JW525781 \\
\hline & hsp20 \#1 & 38.2 & 24.2 & 3 & 3 & JW519178 & JW525783 \\
\hline & hsp70 \#1 & 2.5 & 11 & 1.3 & 6 & JW519142 & JW525772 \\
\hline & hsp70 \#2 & 224 & 95.1 & 7.8 & 19.8 & JW519350 & JW526654 \\
\hline & hsp70 \#3 & 0.7 & 1 & 1.8 & 0.6 & JW506218 & JW525763 \\
\hline & hsp70 \#4 & 0.9 & 0.8 & 1.3 & 0.8 & JW506217 & JW525759 \\
\hline & hsp70 \#5 & 13 & 15 & 3.8 & 5.2 & JW506905 & JW526653 \\
\hline & hsp70 \#15 & 6.1 & 5.2 & 2.6 & 1 & JW502097 & JW521081 \\
\hline & hsp70 \#16 & 2.7 & 1.2 & 1.3 & 1.4 & JW506226 & JW526652 \\
\hline & hsp70 \#17 & 1 & 0.8 & 1.8 & 0.8 & JW506216 & JW525758 \\
\hline & DNAJ \#1 & 1.1 & 0.9 & 2.1 & 1 & JW503763 & JW522979 \\
\hline & DNAJ \#2 & 1.2 & 1 & 1.2 & 1 & JW504839 & JW524198 \\
\hline & DNAJ \#3 & 1.2 & 4.2 & 1 & 2.8 & JW503761 & JW522978 \\
\hline & DNAJ \#4 & 1.1 & 1.3 & 0.5 & 1.5 & JW503762 & JW522980 \\
\hline
\end{tabular}

southern California is more tolerant of thermal stress and exhibited a much larger up-regulation of stressrelated genes following heat shock. In particular, this included a large number of genes that are members of heat shock protein families. Although there is upregulation of $h s p 70$ paralogs in Santa Cruz, the fold change is much smaller than observed in the San Diego population. These results contrast with numerous other studies over latitudinal gradients that have not detected interpopulation expression differences in hsps [26]. For example, even at the interspecific level, Lockwood et al. [5] found that the magnitude of expression of seven hsp70 genes was strikingly similar between a northern and a southern species of Mytilus mussels. Only one $h s p$ ( $h s p 24)$ exhibited a strong difference in expression between the two geographically separated species.

We also examined whether structural variation in Hsp orthologs might explain differential survivorship following acute thermal stress. Sequence analyses of the Hsp gene families suggest population differentiation at the structural level is not elevated compared to the rest of the genome. We identified 42 members of Hsp families across the San Diego and Santa Cruz populations and 29 were assigned to a matching ortholog. While some 
differences are evident in these orthologous copies, the amount of sequence divergence (average $2.9 \%$ ) is close to the median level of $2.7 \%$ sequence divergence found across all genes in a comparison of the San Diego and Santa Cruz transcriptomes [38]. The orthologous pair with the largest amount of sequence divergence in our analysis $(8.7 \%, h s p 67 b 2 \# 1)$ is not an outlier considering the range of sequence divergence evident across the entire transcriptome [38]. Finally, the lack of evidence that non-synonymous substitution rates are elevated in these genes, suggests that positive selection on the coding region may have a limited role in the diversification of heat shock protein function. We note that nonsynonymous substitution rates were measured across entire genes; more rigorous tests of selection based on codon-substitution models will require sampling individuals across multiple populations of $T$. californicus [47].

In addition to the Hsp genes, several other functional categories of genes showed significant response to thermal stress. One similarity between the two populations was the pattern of down-regulation of genes encoded in the mtDNA. Since certain reactions in the electron transport chain are known to be major sources of reactive oxygen species [48], a decrease in expression of mtDNA genes may represent a means of reducing stress. In contrast to the mtDNA genes, most orthologous genes showing significant change in expression in response to thermal stress have diverged either in the magnitude of expression or direction of regulation. Perhaps the most striking difference observed was in genes involved in cuticle formation; these were enriched and up-regulated in San Diego, while being down-regulated in Santa Cruz. $\mathrm{Cu}-$ ticle genes are known to be up-regulated during diapause in many invertebrates [49], presumably to reduce cuticle permeability, but their specific role during acute thermal stress is not known. Genes functioning in proteolysis, which are known to play an important role in protein degradation following stress, also showed a differential response with significant up-regulation of some genes in southern but not in northern populations of Tigriopus. This pattern is opposite to the pattern found in Mytilus [5], where northern populations exhibit an up-regulation of proteolytic genes.

In sum, our results show a variety of significant differences between populations in gene regulation following acute thermal stress and we hypothesize that these contribute to the population differences in survivorship following stress. While mRNA transcript abundance is not equivalent to protein abundance [50], other studies have shown that gene expression changes in experimental treatments are relevant predictors of organismal physiology [51], and that there is often a high correlation between gene expression and protein abundance, including the Hsp gene family [52].

\section{Evidence of local adaptation in Tigriopus californicus}

Numerous species exhibit morphological and life history variation along latitudinal clines [53,54]. These traits typically involve changes in body size, phenology $[11,12,55]$, or temperature adaptation [56]. Recently, Willett [32] and Kelly et al. [35] provided evidence that the intertidal copepod Tigriopus californicus exhibits variation in temperature tolerance along a latitudinal cline in California. Willett showed that populations in northern California exhibit reduced survivorship when exposed to temperatures above $36^{\circ} \mathrm{C}$ for one hour, while survivorship remained high in southern California populations. Additionally, southern California populations exposed to chronic temperature stress survived longer than populations from northern California. Based on observed tradeoffs in fitness across a range of temperatures, Willett argued that this provided evidence of local adaptation to temperature in T. californicus. Kelly et al. [35] used selection experiments to demonstrate that thermal tolerance of $T$. californicus responded to selection over 10 generations, providing direct evidence for additive genetic variation for tolerance to thermal stress. Furthermore, Kelly et al. found that total quantitative genetic variation was almost completely partitioned among populations, rather than within populations, suggesting strong local adaptation.

Our results support the conclusions of both of the above studies and provide a possible molecular basis for local adaptation, namely that the pathways that control the regulation of numerous genes involved in response to acute temperature stress have diverged. Other studies have shown that stabilizing selection often acts to prevent genetic differentiation in expression patterns among populations and among species $[57,58]$. The clearest pattern in our study emerges from the dramatic differences in expression in the $h s p 70$ gene. Heat shock genes are known to be important components of temperature adaptation and resistance to thermal stress [1,59], as they play a critical role in preventing the degradation of intracellular proteins. Whole transcriptome studies have shown that Hsps play a dominant role in heat stress response in other species [60], as well as responding to other forms of environmental stress $[61,62]$. Rapid adaptation to novel thermal environments by altering expression of Hsps, and that of other chaperone proteins, may be a common form of local population adaptation in diverse taxa [5].

\section{Conclusions}

In this study we contrasted the whole genome response in gene expression between two T. californicus populations that exhibit differences in tolerance to thermal stress. Broad changes in gene expression were observed, with the more thermal tolerant San Diego population 
showing more pronounced up-regulation of $h s p$ and ubiquitin pathway genes than the more sensitive Santa Cruz population. Although this up-regulation involves a large number of heat shock proteins, the most dramatic response involved the $h s p 70$ gene family. Orthologous copies of the Hsps do not show evidence of divergent selection for structural changes, suggesting, at least in part, that regulatory variation between populations may play an important role in their differential sensitivity to acute heat stress.

\section{Additional files}

Additional file 1: Table S1. List of genes and primers examined in quantitative PCR trials of Tigriopus californicus.

Additional file 2: Figure S1. Smoothed density estimates of the reads per kilobase per million reads (RPKM) of contigs in each treatment for A) the whole transcriptome and B) significantly differentially expressed genes.

Additional file 3: Table S2. Gene ontology terms (F: function, P: process, C: cellular component) for significantly up or down-regulated genes.

\section{Competing interests}

The authors' have no competing interests to declare.

\section{Authors' contributions}

SDS carried conducted the heat shock treatments, carried out the transcriptome analysis, and drafted the manuscript. FSB assisted in the transcriptome assembly, QPCR analysis and helped to draft the manuscript. GWM prepared the transcriptome samples. AW sequenced the HSP genes and assisted with GPCR analysis. RSB initiated the design and coordination of the study, obtained funding, and helped to draft the manuscript. All authors read and approved the final manuscript.

\section{Acknowledgements}

We would like to acknowledge The Scripps Research Institute DNA Array Core Facility for conducting the Illumina Sequencing. We would also like to thank the anonymous reviewers for their helpful comments. This study was funded in part by grants from the National Science Foundation (DEB 0717178, DEB 1051057) to RSB.

\section{Author details}

${ }^{1}$ Marine Biology Research Division, Scripps Institution of Oceanography, University of California, San Diego, 9500 Gilman Drive, La Jolla, CA 92093-0202, U.S.A. ²Département de Biologie, Ecole Normale Supérieure de Paris, 46, rue d'Ulm, 75005, Paris, France.

Received: 2 March 2012 Accepted: 30 August 2012

Published: 5 September 2012

\section{References}

1. Angilletta MJ: Thermal adaptation: a theoretical and empirical synthesis. Oxford: Oxford University Press; 2009.

2. Clarke $A$ : Costs and consequences of evolutionary temperature adaptation. Trends Ecol Evol 2003, 18:573-581.

3. Davenport J, Davenport JL: Effects of shore height, wave exposure and geographical distance on thermal niche width of intertidal fauna. Mar Ecol Prog Ser 2005, 292:41-50.

4. Sorte CJB, Hofmann GE: Changes in latitude, changes in aptitudes: Nucella canaliculata (Mollusca: Gastropoda) is more stressed at its range edge. Mar Ecol Prog Ser 2004, 274:263-268.
5. Lockwood BL, Sanders JG, Somero GN: Transcriptomic responses to heat stress in invasive and native blue mussels (genus Mytilus): molecular correlates of invasive success. J Exp Biol 2010, 213:3548-3558.

6. Kuo ESL, Sanford E: Geographic variation in the upper thermal limits of an intertidal snail: implications for climate envelope models. Mar Ecol Prog Ser 2009, 388:137-146.

7. James AC, Azevedo RBR, Partridge L: Genetic and environmental responses to temperature of Drosophila melanogaster from a latitudinal cline. Genetics 1997, 146:881-890.

8. Ayrinhac A, Debat V, Gibert P, Kister A-G, Legout H, Moreteau B, Vergilino R, David JR: Cold adaptation in geographical populations of Drosophila melanogaster: phenotypic plasticity is more important than genetic variability. Funct Ecol 2004, 18:700-706.

9. Kawecki TJ, Ebert D: Conceptual issues in local adaptation. Ecol Lett 2004, 7:1225-1241.

10. Lenormand T: Gene flow and the limits to natural selection. Trends Ecol Evol 2002, 17:183-189.

11. Li Y, Huang Y, Bergelson J, Nordborg M, Borevitz JO: Association mapping of local climate-sensitive quantitative trait loci in Arabidopsis thaliana. Proc Natl Acad Sci USA 2010, 107:21199-21204.

12. Ikeda $\mathrm{H}$, Setoguchi $\mathrm{H}$ : Natural selection on PHYE by latitude in the Japanese archipelago: insight from locus specific phylogeographic structure in Arcterica nana (Ericaceae). Mol Ecol 2010, 19:2779-2791.

13. Storz J, Sabatino S, Hoffmann F, Gering E, Moriyama H, Ferrand N, Monteiro B, Nachman M: The molecular basis of high-altitude adaptation in deer mice. PLoS Genet 2007, 3:e45.

14. Dahlhoff EP, Rank NE: Functional and physiological consequences of genetic variation at phosphoglucose isomerase: Heat shock protein expression is related to enzyme genotype in a montane beetle. Proc Natl Acad Sci USA 2000, 97:10056-10061.

15. Hoekstra HE, Drumm KE, Nachman MW: Ecological genetics of adaptive color polymorphism in pocket mice: geographic variation in selected and neutral genes. Evolution 2004, 58:1329-1341.

16. Schulte PM: Environmental adaptations as windows on molecular evolution. Comp Bioch Phys B 2001, 128:597-611.

17. Arnold FH, Wintrode PL, Miyazaki K, Gershenson A: How enzymes adapt: lessons from directed evolution. Trends Biochem Sci 2001, 26:100-106.

18. McDonald JF, Chambers GK, David J, Ayala FJ: Adaptive response due to changes in gene regulation: a study with Drosophila. Proc Natl Acad Sci USA 1977, 74:4562-4566.

19. Barrier M, Robichaux RH, Purugganan MD: Accelerated regulatory gene evolution in an adaptive radiation. Proc Natl Acad Sci USA 2001, 98:10208-10213.

20. Carroll SB: Endless forms: the evolution of gene regulation and morphological diversity. Cell 2000, 101:577-580.

21. Hofmann G, Somero G: Evidence for protein damage at environmental temperatures: seasonal changes in levels of ubiquitin conjugates and hsp70 in the intertidal mussel Mytilus trossulus. J Exp Biol 1995, 198:1509-1518.

22. Hofmann G, Somero G: Interspecific variation in thermal denaturation of proteins in the congeneric mussels Mytilus trossulus and $M$. galloprovincialis: evidence from the heat-shock response and protein ubiquitination. Mar Biol 1996, 126:65-75.

23. Sagarin RD, Somero GN: Complex patterns of expression of heat-shock protein 70 across the southern biogeographical ranges of the intertidal mussel Mytilus californianus and snail Nucella ostrina. J Biogeogr 2006, 33:622-630.

24. Dutton JM, Hofmann GE: Biogeographic variation in Mytilus galloprovincialis heat shock gene expression across the eastern Pacific range. J Exp Mar Biol Ecol 2009, 376:37-42.

25. Hammond LM, Hofmann GE: Thermal tolerance of Strongylocentrotus purpuratus early life history stages: mortality, stress-induced gene expression and biogeographic patterns. Mar Biol 2010, 157:2677-2687.

26. Feder ME, Hofmann GE: Heat-shock proteins, molecular chaperones, and the stress response: evolutionary and ecological physiology. Annu Rev Physiol 1999, 61:243-282

27. Gracey AY, Chaney ML, Boomhower JP, Tyburczy WR, Connor K, Somero GN Rhythms of gene expression in a fluctuating intertidal environment. Curr Biol 2008, 18:1501-1507. 
28. Place SP, O'Donnell MJ, Hofmann GE: Gene expression in the intertidal mussel, Mytilus californianus: physiological response to environmental factors on a biogeographic scale. Mar Ecol Prog Ser 2008, 356:1-14.

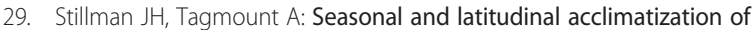
cardiac transcriptome responses to thermal stress in porcelain crabs, Petrolisthes cinctipes. Mol Ecol 2009, 18:4206-4226.

30. Wang Z, Gerstein M, Snyder M: RNA-Seq: a revolutionary tool for transcriptomics. Nat Rev Genet 2009, 10:57-63.

31. Mortazavi A, Williams BA, McCue K, Schaeffer L, Wold B: Mapping and quantifying mammalian transcriptomes by RNA-Seq. Nat Meth 2008 5:621-628.

32. Willett CS: Potential fitness trade-offs for thermal tolerance in the intertidal copepod Tigriopus californicus. Evolution 2010, 64:2521-2534.

33. Powlick JJ: Habitat characters of Tigriopus californicus (Copepoda: Harpacticoida), with notes on the dispersal of supralittoral fauna. J Mar Biol Assoc UK 1999, 79:85-92.

34. Edmands S, Deimler JK: Local adaptation, intrinsic coadaptation and the effects of environmental stress on interpopulation hybrids in the copeod Tigriopus californicus. J Exp Mar Biol Ecol 2004, 303:183-196.

35. Kelly MW, Sanford E, Grosberg RK: Limited potential for adaptation to climate change in a broadly distributed marine crustacean. Proc $R$ Soc Lond B Biol Sci 2012, 279:349-356.

36. Kendziorski C, Irizarry RA, Chen KS, Haag JD, Gould MN: On the utility of pooling biological samples in microarray experiments. Proc Natl Acad SC USA 2005, 102:4252-4257.

37. Marioni JC, Mason CE, Mane SM, Stephens M, Gilad Y: RNA-seq: An assessment of technical reproducibility and comparison with gene expression arrays. Genome Res 2008, 18:1509-1517.

38. Barreto FS, Moy GW, Burton RS: Interpopulation patterns of divergence and selection across the transcriptome of the copepod Tigriopus californicus. Mol Ecol 2011, 20:560-572.

39. Conesa A, Götz S, García-Gómez JM, Terol J, Talón M, Robles M: Blast2GO: a universal tool for annotation, visualization and analysis in functional genomics research. Bioinformatics 2005, 21:3674-3676.

40. Götz S, García-Gómez JM, Terol J, Williams TD, Nagaraj SH, Nueda MJ, Robles M, Talón M, Dopazo J, Conesa A: High-throughput functional annotation and data mining with the Blast2GO suite. Nucleic Acids Res 2008, 36:3420-3435.

41. The Gene Ontology Consortium, Ashburner M, Ball CA, Blake JA, Botstein D, Butler H, Cherry JM, Davis AP, Dolinski K, Dwight SS, et al: Gene Ontology: tool for the unification of biology. Nat Genet 2000, 25:25-29.

42. Kal AJ, van Zonneveld AJ, Benes V, van den Berg M, Koerkamp MG, Albermann K, Strack N, Ruijter JM, Richter A, Dujon B, et al: Dynamics of gene expression revealed by comparison of serial analysis of gene expression transcript profiles from yeast grown on two different carbon sources. Mol Biol Cell 1999, 10:1859-1872.

43. Miyata T, Yasunaga T: Molecular evolution of mRNA: a method for estimating evolutionary rates of synonymous and amino acid substitutions from homologous nucleotide sequences and its applications. J Mol Evol 1980, 16:23-36.

44. Larkin MA, Blackshields G, Brown NP, Chenna R, McGettigan PA, McWilliam $H$, Valentin F, Wallace IM, Wilm A, Lopez R, et al: Clustal W and Clustal X version 2.0. Bioinformatics 2007, 23:2947-2948.

45. Tamura K, Peterson D, Peterson N, Stecher G, Nei M, Kumar SF: MEGA5: molecular evolutionary genetics analysis using maximum likelihood, evolutionary distance, and maximum parsimony methods. Mol Biol Evol 2011, 28:2731-2739.

46. Vandesompele J, De Preter K, Pattyn F, Poppe B, Van Roy N, De Paepe A, Speleman F: Accurate normalization of real-time quantitative RT-PCR data by geometric averaging of multiple internal control genes. Genome Biol 2002, 3:research0034.0031-research0034.0011.

47. Schoville SD, Flowers JM, Burton RS: Diversifying selection underlies the origin of allozyme polymorphism at the phosphoglucose isomerase locus in Tigriopus californicus. PLoS One 2012, 7:e40035.

48. Murphy MP: How mitochondria produce reactive oxygen species. Biochem J 2009, 417:1-13.

49. MacRae T: Gene expression, metabolic regulation and stress tolerance during diapause. Cell Mol Life Sci 2010, 67:2405-2424.

50. Feder ME, Walser JC: The biological limitations of transcriptomics in elucidating stress and stress responses. J Evol Biol 2005, 18:901-910.
51. Maier T, Güell M, Serrano L: Correlation of mRNA and protein in complex biological samples. FEBS Lett 2009, 583:3966-3973.

52. Buckley BA, Gracey AY, Somero GN: The cellular response to heat stress in the goby Gillichthys mirabilis: a cDNA microarray and protein-level analysis. J Exp Biol 2006, 209:2660-2677.

53. Endler JA: Geographic Variation, Speciation, and Clines. Princeton, NJ: Princeton University Press; 1977.

54. Vermeij GJ: Biogeography and Adaptation: Patterns of Marine Life. Cambridge: Mass.: Harvard University Press; 1978.

55. Ragland GJ, Kingsolver JG: Influence of seasonal timing on thermal ecology and thermal reaction norm evolution in Wyeomyia smithii. J Evol Biol 2007, 20:2144-2153.

56. Sarup P, Frydenberg J, Loeschcke V: Local adaptation of stress related traits in Drosophila buzzatii and Drosophila simulans in spite of high gene flow. J Evol Biol 2009, 22:1111-1122.

57. Lemos B, Meiklejohn CD, Cáceres M, Hartl DL: Rates of divergence in gene expression profiles of primates, mice, and flies: stabilizing selection and variability among functional categories. Evolution 2005, 59:126-137.

58. Whitehead A, Crawford DL: Variation within and among species in gene expression: raw material for evolution. Mol Ecol 2006, 15:1197-1211.

59. Sørensen JG, Kristensen TN, Loeschcke V: The evolutionary and ecological role of heat shock proteins. Ecol Lett 2003, 6:1025-1037.

60. Sørensen JG, Nielsen MM, Kruhøffer M, Justesen J, Loeschcke V: Full genome gene expression analysis of the heat stress response in Drosophila melanogaster. Cell Stress Chaperon 2005, 10:312-328.

61. Chapman RW, Mancia A, Beal M, Veloso A, Rathburn C, Blair A, Holland AF, Warr GW, Didinato GUY, Sokolova IM, et al: The transcriptomic responses of the eastern oyster, Crassostrea virginica, to environmental conditions. Mol Ecol 2011, 20:1431-1449.

62. Rhee J-S, Raisuddin S, Lee K-W, Seo JS, Ki J-S, Kim I-C, Park HG, Lee J-S: Heat shock protein $(\mathrm{Hsp})$ gene responses of the intertidal copepod Tigriopus japonicus to environmental toxicants. Comp Biochem Phys C 2009, 149:104-112.

doi:10.1186/1471-2148-12-170

Cite this article as: Schoville et al:: Investigating the molecular basis of local adaptation to thermal stress: population differences in gene expression across the transcriptome of the copepod Tigriopus californicus. BMC Evolutionary Biology 2012 12:170.

\section{Submit your next manuscript to BioMed Central and take full advantage of:}

- Convenient online submission

- Thorough peer review

- No space constraints or color figure charges

- Immediate publication on acceptance

- Inclusion in PubMed, CAS, Scopus and Google Scholar

- Research which is freely available for redistribution 